\title{
Research on Fault Distribution Parameters Estimation and Reliability Assessment of CNC Grinding Machine Tools
}

\author{
Jinwei Fan ${ }^{1, a}$, Shi $\mathrm{Ji}^{2, \mathrm{~b}}$ and Shuai Yuan ${ }^{2, \mathrm{c}}$ \\ ${ }^{1}$ School of Mechanical Engineering, Beijing University of Technology, Beijing 100124, China; \\ ${ }^{2}$ School of Mechanical Engineering, Beijing University of Technology, Beijing 100124, China. \\ ajwfan@bjut.edu.cn, ${ }^{\mathrm{b}} 1469578848 @ q q . c o m,{ }^{\mathrm{c}}$ 530211126qq.com
}

Keywords: Reliability; Probability Density Function; Least Square Method; Parameter Estimation; MTBF; CNC

\begin{abstract}
Fault distribution type identification and probability density function parameters estimation are the prerequisite of CNC grinding machine tools' reliability assessment. The procedure of fault data grouping, the technique of probability density function parameters estimation based on least square method and the method of reliability index (mean time between failures, MTBF ) calculation are proposed. The probability density function parameters estimation and curve fitting, the comparison of goodness of fit, the MTBF solution of some type crankshaft are carried out by the method proposed in the paper. The results of statistical calculation and direct calculation by definition are close. The procedure of reliability assessment proposed here can be programmed easily and it provides one measure for reliability assessment of CNC grinding machine tools.
\end{abstract}

\section{Introduction}

In the case of technical innovation is more and more difficult, product competitiveness is the key .The reliability of the high and low. Improve product reliability is the premise of establishing fault probability density of the product .Degrees of function, determine product failure distribution types, evaluate the reliability of the current level. On product reliability evaluation methods both at home and abroad to carry out a large amount of research, has obtained certain the research results. Samanta B by using the fault tree of minimum cut set method is proposed reliability evaluation [1], this method need to set up the fault tree of the product, the efficiency is low. Yang Na waiting for qualitative and quantitative methods of mechanical system reliability are studied, but not on the calculation of reliability index [2].Index [3-4] use monte carlo simulation to evaluate the reliability of the product, the evaluation method of accuracy depends on the correctness of the prior distribution function, and calculate the cost.Index [5-6] proposed the use of bayesian network to evaluate the reliability of the products, the establishment of the bayesian network workload is big, and if missing it is hard to guarantee the correctness of the calculation results.Index[7] using genetic algorithm to solve the failure distribution function parameter. At present, the research on reliability evaluation methods focus on aerospace and military equipment, the reliability research of common mechanical and electrical equipment are relatively few. Based on this background, the nc grinding machine failure data statistics, the types of common faults distribution, the distribution of all kinds of parameter estimation and reliability calculation, etc are studied, put forward from the failure data processing to the failure distribution fitting and the reliability index calculation method. Described method can facilitate the formation of a computer program, convenience is provided for evaluating the reliability of CNC grinding machine. 


\section{Organization of the Text}

\section{Determine the fault type of distribution}

Failure data statistics. To discover the failure distribution of mechanical and electrical products, and then evaluate the reliability of the product level, must be conducted on product reliability test, fault data. In order to enhance the reliability of fault data, the failure data must be collected enough, often carrying out reliability test of timing truncation. After get the reliability data, the failure data are grouped according to time, the size of the number of units to the right. Group number is too small, cannot really reflect the subsystem failure probability density, the group number is too large, increase the amount of calculation, the fitting is difficult. The number of group size can be made of type (1) to calculate [8].

$$
\hat{\mathrm{k}}=1+3.3 \log \mathrm{n}_{\mathrm{T}}
$$

\section{$n_{T}$-timing truncated total failure for a set period of time}

According to the type (1) after calculation and out, the roundness is to get the number of clusters $\mathrm{k}$. In order to ensure the accuracy of the fitting, if the calculated value of $\mathrm{k}$ is less than 8 , can take to 8. After calculating $\mathrm{k}$ value, the cut-off time are divided into $\mathrm{k}$ range, number of fault statistics each time interval mapping tables, as shown in table 1.

Table 1 failure data packet

\begin{tabular}{ccccc}
\hline No. & $t_{i-}$ & $t_{i+}$ & $t_{i}$ & $n_{i}$ \\
\hline 1 & 0 & $T / k$ & $T / 2 k$ & $n_{1}$ \\
2 & $T / k$ & $2 T / k$ & $3 T / 2 k$ & $n_{2}$ \\
$\cdots$ & $\cdots$ & $\cdots$ & $\cdots$ & $\cdots$ \\
$\mathrm{k}$ & $(K-1) T / k$ & $\mathrm{~T}$ & $(2 k-1) T / 2 k$ & $n_{k}$
\end{tabular}

In the table: $\mathbf{t}_{\mathbf{i}-}$-Packet interval of the left endpoint

$\mathbf{t}_{\mathbf{i}}$-The middle of the packet interval value

$\mathbf{t}_{\mathbf{i}+-}$-Packet interval right endpoints

$\mathbf{n}_{i}-$ The statistics on the number of fault in the group $i$

Determine the fault type of distribution. After the failure of each interval number, can use type (2) the observation of each interval failure probability density values.

$$
\hat{f}\left(t_{i}\right)=\frac{n_{i}}{t n_{t}}
$$

After the failure of each interval number, can use type (2) the observation of each interval failure probability density values.

\section{Parameter estimation}

The least square method. After preliminary determine the fault distribution type, need to estimate the parameters of failure probability density function. Because of simple, least-square method is one of the commonly used method in parameter estimation [9], the basic idea is to assume that there are $\mathrm{n}$ observations $\{\mathrm{xi}, \mathrm{yi}\}(\mathrm{I}=1,2, \ldots, \mathrm{n})$, if there is a linear relation between $\mathrm{x}$ and $\mathrm{y}$, then you can use straight lines to fitting the $\mathrm{x}, \mathrm{y}$, the change of the relationship between such as shown in (3).

$$
\hat{y}=a \hat{x}+b
$$

Using the least square method can calculate the parameter type (3) a and b values, as shown in type (4).

$$
\left\{\begin{array}{c}
\mathrm{a}=\frac{1}{\mathrm{n}} \sum_{\mathrm{i}=1}^{\mathrm{n}} \mathrm{y}_{\mathrm{i}}-\frac{6}{\mathrm{n}} \sum_{\mathrm{i}=1}^{\mathrm{n}} \mathrm{x}_{\mathrm{i}} \\
\mathrm{b}=\frac{\sum_{\mathrm{i}=1}^{\mathrm{n}}\left(\mathrm{x}_{\mathrm{i}}-\frac{1}{\mathrm{n}} \sum_{\mathrm{i}=1}^{\mathrm{n}} \mathrm{x}_{\mathrm{i}}\right)\left(\mathrm{y}_{\mathrm{i}}-\frac{1}{\mathrm{n}} \sum_{\mathrm{i}=1}^{\mathrm{n}} \mathrm{y}_{\mathrm{i}}\right)}{\sum_{\mathrm{i}=1}^{\mathrm{n}}\left(\mathrm{x}_{\mathrm{i}}-\frac{1}{\mathrm{n}} \sum_{\mathrm{i}=1}^{\mathrm{n}} \mathrm{x}_{\mathrm{i}}\right)}
\end{array}\right.
$$

To take advantage of the least square method to fault distribution function parameter estimates, you need to construct linear equation, by type (4) is used to estimate the values of a and b, reverse the parameters in the probability density function. So structure linear relation is the key point of using the least squares method for parameter estimation. Below is for the CNC grinding machine two of the most common fault distribution to construct linear equation, then the parameter estimation of failure distribution function.

An index distribution.The cumulative distribution function with exponential distribution of 
expression, as shown in the type (5) there is a $\chi$.

$$
\mathrm{F}(\mathrm{t})=1-\mathrm{e}^{-\lambda \mathrm{t}}
$$

To (5) on both sides of the exponential type, available type (6) ,cause $y=\ln ;\left[1-F\left(t \_i\right) ~\right], x=t \_i, s o$ $y=-\chi_{x}$, if the sample data to satisfy exponential distribution,so the linear relationship. The parameters of the exponential distribution $\chi=-\mathrm{a}$.

$$
\ln \left[1-F\left(t_{i}\right)\right]=-\lambda t_{i}
$$

The type (6) shows that if the fault data meet the exponential distribution, a linear function of its structure should be through the origin, the $b=0$. Obviously, because of the failure data statistics is random, they cannot guarantee the linear structure just the origin, the structure of linear equation can be modified at this time. As shown in figure 1, figure in implementing line for fitting curve, if the curve does not pass through the origin, the need to modify.

Figure 1, the length of the oc for the value of $b$ is the least square method, in order to make the fitting curve by origin, this time can rotate an Angle, an $d$ the dotted line in figure 1. Usually, if the statistics meet exponential distribution, the value of $b$ is very small, at this point a revised can be calculated by the type (7).

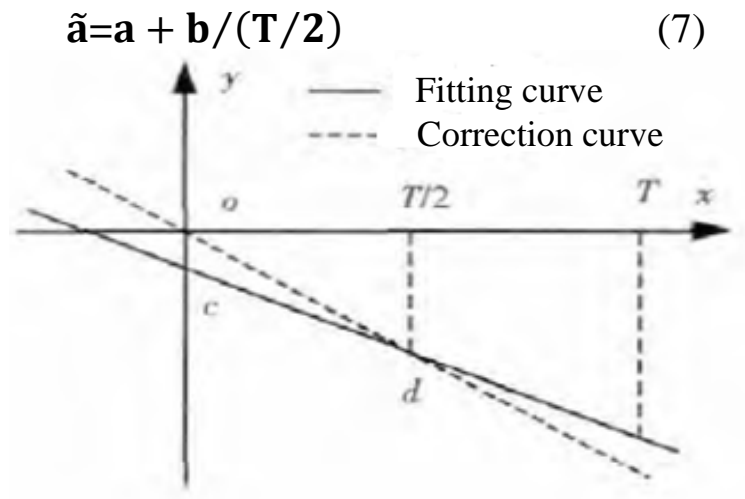

Fig.1 Modification of Exponential Distribution

Weibull distribution. The cumulative distribution expressions of two parameter weibull distribution as shown in type (8).

$$
\mathrm{F}\left(t_{i}\right)=1-\exp \left|-\left(t_{i} / \eta\right)^{m}\right|
$$

Will type (8) on both sides of the exponential twice,cause $y=\ln \ln \frac{1}{1-F\left(t_{i}\right)}, x=\ln t_{i}$,so $y=m s-m \ln \eta$. If $t_{i}$ obey weibull distribution,so is a linear relationship, using the least square method can calculate weibull distribution parameters of $m$ and $\eta$, as shown in type (9).

$$
\left\{\begin{array}{c}
\widehat{m}=b \\
\hat{y}=\exp \left(-\frac{a}{b}\right)
\end{array}\right.
$$

The test of goodness of fit.If you find fault distribution function of scatter plot conform to the distribution of two or more, you need to determine what kind of distribution and statistical data, better at this time can compare of goodness of fit. Goodness of fit with the $\mathrm{R}$ value, as shown in type (10).

Type (10), $\mathrm{y}_{i}$ is fitting values, $\widehat{y}_{l}$ was observed value, $\bar{y}$ is the average of the $\mathrm{y}_{i}$. $\mathrm{R}$, the more close to 1 , indicating the better fitting effect. When fitting curve satisfy multiple distribution type, you can compare the goodness of fit value, take the goodness-of-fit curve of maximum value as the fitting results.

\section{Reliability evaluation}

$$
R^{2}=\frac{\sum_{i=1}^{n}\left(y_{i}-\bar{y}\right)^{2}}{\sum_{i=1}^{n}\left(\hat{y}_{i}-\bar{y}\right)^{2}}
$$

The product parameter estimation and fault probability density curve fitting to assess the reliability of the products is the purpose of the index MTBF, its definition as shown in type (11).

$$
\mathrm{MTBF}=\int_{0}^{\infty} t f(t) d t
$$

Exponential distribution and weibull distribution is presented to solve reliability MTBF value such as type (12) type as shown in (13). 


$$
\begin{aligned}
\operatorname{MTBF}_{e} & =\frac{1}{\lambda} \\
\operatorname{MTBF}_{\mathrm{U}} & =\operatorname{\eta у\Gamma }\left(1+\frac{1}{\mathrm{~m}}\right)
\end{aligned}
$$

\section{Summary}

Analysis of the CNC machine tool failure probability distribution of common types, puts forward to determine the fault distribution types of steps and methods of mechanical and electronic products, introduces the failure probability density function to make use of least square parameter estimation method. The proposed method can easily write a computer program is used to estimate parameters and the reliability index is calculated. (2) using the proposed method of reliability assessment and reliability refers to the direct use of the definition of MTBF solving relatively close, the reliability of the proposed evaluation method to evaluate the reliability index of the mechanical and electrical products, provides reference for reliability design.

\section{References}

[1] B.Samanta, B.Sarkar, S.K.Mukherjee.Reliability assessment of hydraulic shovel system using fault trees $[\mathrm{J}]$.Transactions of the Institutions of Mining and Metallurgy, Section A:Mining Technology, 2002 (111) : 129-135.

[2] Yang Na, Ye Guo-ming.Summary of qualitative and quantitative design methods on machinery reliability $[\mathrm{J}]$.Machinery Design \& Manufacture, 2006 (3) : 16-19.

[3] L.Goel, X.Liang, Y.Ou.Monte Carlo simulation -based customer service reliability assessment [J] .Electric Power Systems Research, 1999, 49 (3) : 185-194.

[4] (Feng Chang -you, Wang Xi -fan, Bie Zhao -hong.Unit maintenance scheduling model based on system reliability $[\mathrm{J}]$.Journal of Xi'an Jiaotong University, 2009, 43（8）: 80-85.)

[5] C.Y.Li, M.Q.Xu, S.Guo.Real-time reliability assessment based on gamma process and bayesian estimation [J].Journal of Astronautics, 2009, 30 (4) : 1722-1726.

[6] ( Xu Ge-ning, Li Yin-de, Yang Heng.Reliability assessment of hydraulic systems in automobile crane based on Bayesian networks [J] .China Safety Science Journal, 2011, 21(5): 90-96.)

[7] G.Yuriy, N.Vayenas.Discrete-event simulation of mine equipment systems combined with a reliability assessment model based on genetic algorithms $[\mathrm{J}]$.International Journal of Mining, Reclamation and Environment, 2008, 22 (1) : 70-83.

[8] (Xue Yu-xia.Research on availability key technique for numerical control machine tools [D] .Changchun: Jilin University, 2009.)

[9] S.C.Kang, H.M.Koh, J.F.Choo.An efficient response surface method using moving least squares approximation for structural reliability analysis $[\mathrm{J}]$. Probabilistic Engineering Mechanics, 2010, 25 (4) : 365-371. 